\title{
Editorial
}

\section{Modeling and Simulation in Transportation Engineering 2014}

\author{
Wuhong Wang, ${ }_{1}^{1}$ Geert Wets, ${ }^{2}$ Heiner Bubb, ${ }^{3}$ Huimin Niu, ${ }^{4}$ and Hong Chen ${ }^{5}$ \\ ${ }^{1}$ Department of Transportation Engineering, Beijing Institute of Technology, Beijing 100081, China \\ ${ }^{2}$ Transportation Research Institute (IMOB), Hasselt University, 3590 Diepenbeek, Belgium \\ ${ }^{3}$ Institute of Ergonomics, Technical University of Munich, 85747 Munich, Germany \\ ${ }^{4}$ School of Traffic and Transportation, Lanzhou Jiaotong University, Lanzhou 730070, China \\ ${ }^{5}$ School of Highway, Chang'an University, Xian 710064, China \\ Correspondence should be addressed to Wuhong Wang; wangwuhong@bit.edu.cn
}

Received 17 December 2014; Accepted 17 December 2014

Copyright (C) 2015 Wuhong Wang et al. This is an open access article distributed under the Creative Commons Attribution License, which permits unrestricted use, distribution, and reproduction in any medium, provided the original work is properly cited.

Theoretical investigation and empirical work trigger the industry development and vice versa. In transportation engineering, a proliferation of researches related to each essential element of transportation systems prompt the evolutions at different levels of transportation science and technique. Researchers have been pursuing a safe, intelligent, green transportation system and solving the current problems worldwide. All actions are based on a continuous analysis of revealing a variety of static and dynamic transportation phenomena.

Modeling and simulation are well-established methods for understanding, characterizing, and predicting the aforesaid phenomena (microscopic, mesoscopic, and macroscopic). Modeling addresses how something behaves in an abstractive way. And simulation is an alternative representation as well as a tool set in experimental testing, training, and decision making. This research field has been involved in mathematical, physical, and psychological science and moves forward to an interdisciplinary combination. Nowadays, the studies on mathematical modeling and computational simulation in transportation engineering have significant contributions to the development of green intelligent transportation and safety.

Considering the interaction between traffic user activities and the traffic environment, more efforts need to be made by balancing the human factor and nature ecology. Meanwhile, with the booming travel demand in metropolitan areas, reduction of the traffic congestion should be highly emphasized. Transportation modeling and simulation application integrated with new technologies can be regarded as the foundation work for seeking the solutions to a better sustainable mobility.

The special issue gives an overview of the sophisticated traffic models and discusses an in-depth study of innovative methodologies in modeling and simulation of transportation operations. The proposed novel models and simulators in traffic flow, road user behavior, vehicle safety performance, public transit analysis, transportation policy, and construct measures can be widely applied in transportation planning, design, optimization, operation, and management.

You will be able to find out the advances in modeling and simulation of transportation system. Herewith the main topics of the special issue include the following: intelligent transportation system (ITS), logistics and transportation management, transit and rail systems operation, traffic flow and transportation model, mathematical optimization in traffic engineering, traffic safety and emergence response, renewable energy sources in transportation, transport planning and road traffic, vehicle active safety and intelligent vehicle, and driving behavior and driver assistance system.

Wuhong Wang Geert Wets

Heiner Bubb Huimin Niu Hong Chen 


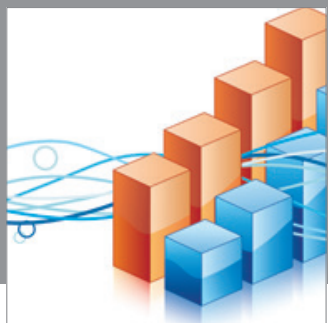

Advances in

Operations Research

mansans

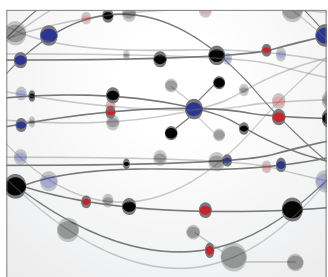

The Scientific World Journal
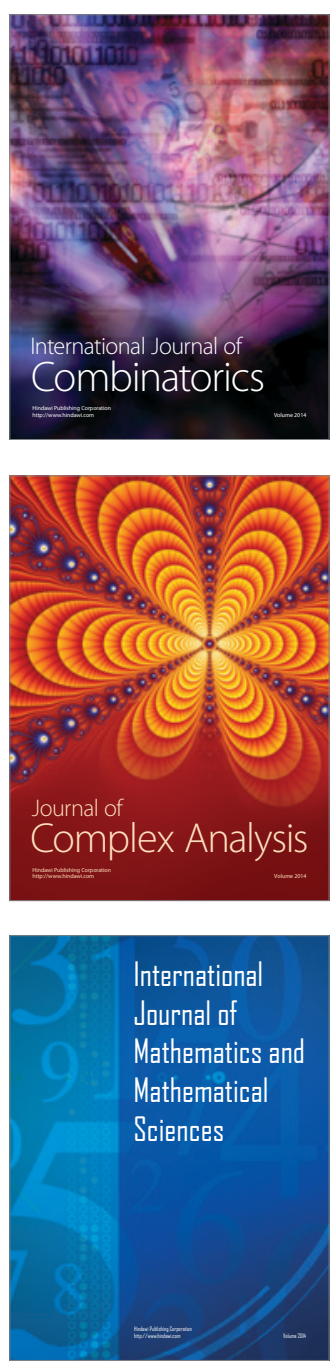
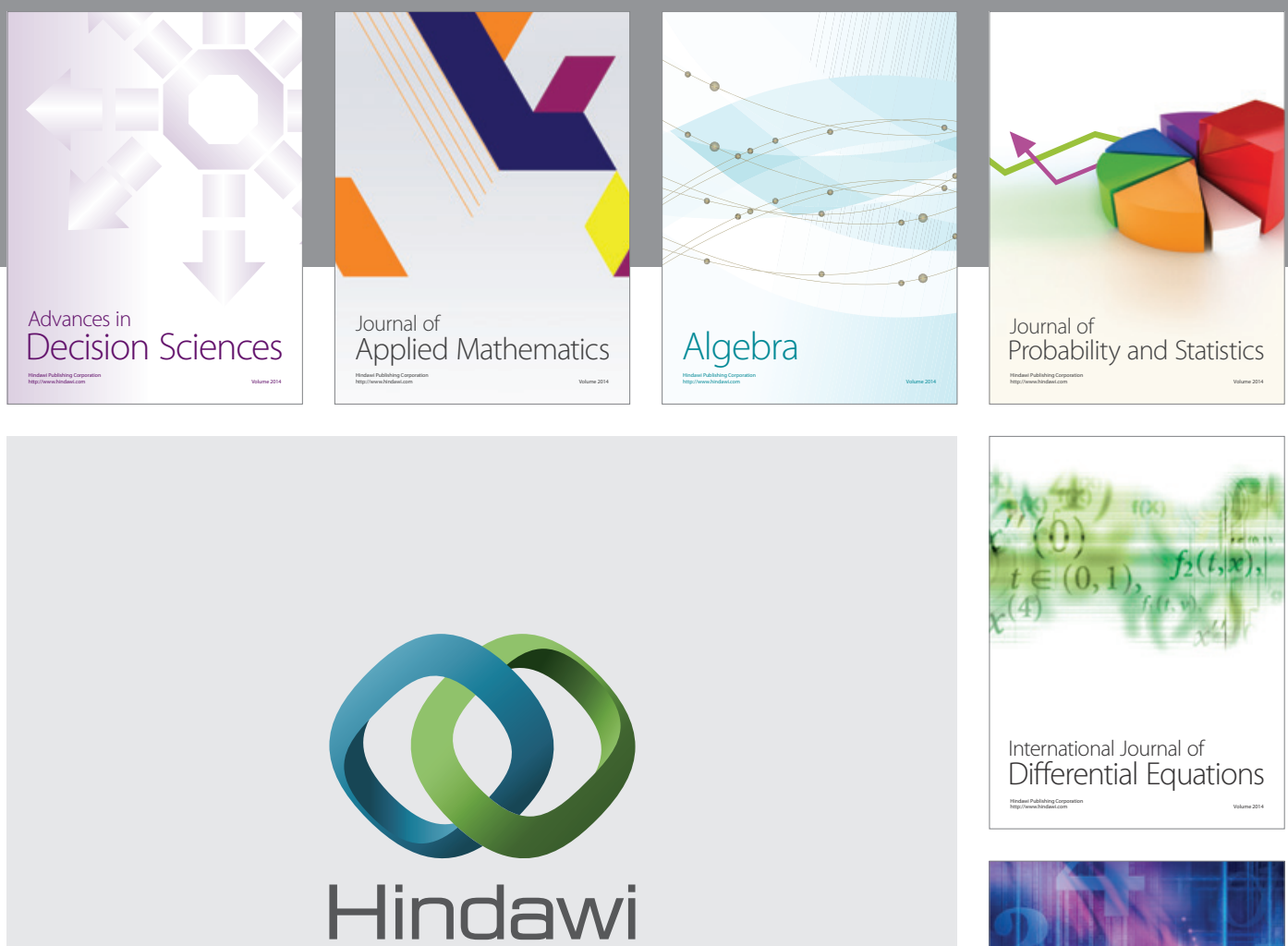

Submit your manuscripts at http://www.hindawi.com
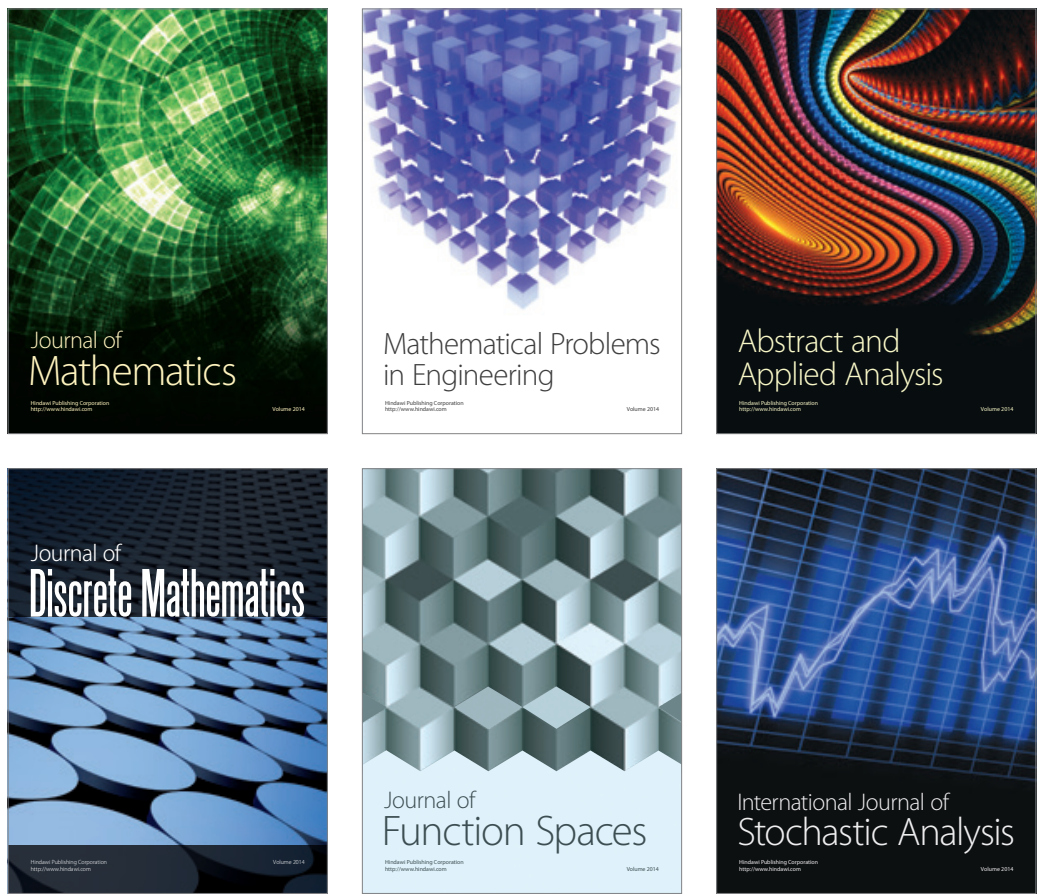

Journal of

Function Spaces

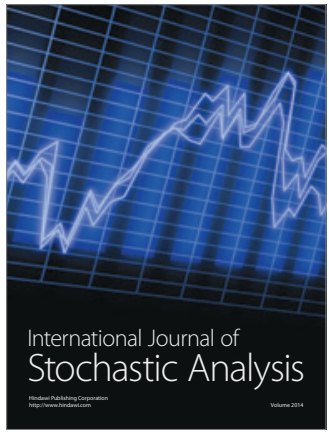

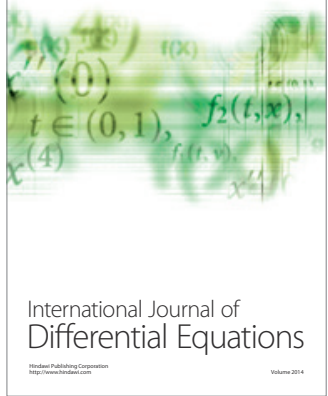
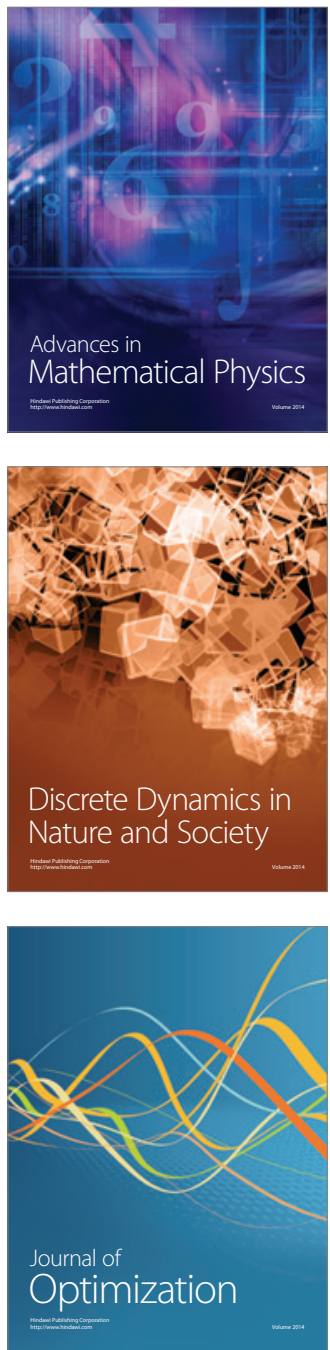